\title{
SISTEMA DE BENCHMARKING COMO ESTRATEGIA DE INNOVACIÓN TECNOLÓGICA EN EL SECTOR CRUDOS PESADOS Y EXTRAPESADOS
}

\author{
Junior Nava Zambrano ${ }^{1} *$ \\ ${ }^{1}$ Petróleos de Venezuela. Maracaibo, Venezuela \\ *Autor de correspondencia: navajar@pdvsa.com
}

Recibido Octubre 2020; Aceptado Diciembre 2020

\begin{abstract}
Resumen - El objetivo general del trabajo presentado consistió en proponer un plan estratégico basado en el benchmarking como estrategia de innovación tecnológica para el sector crudos pesados y extrapesados. La investigación fue de tipo explicativa, de campo, con modalidad proyecto factible, con un diseño no experimental y transeccional descriptivo, la muestra correspondió a seis empresas mixtas del sector crudos pesados y extrapesados, seleccionadas mediante muestreo no probabilístico basado en criterios de selección. La investigación se desarrolló mediante la técnica de encuesta, con un cuestionario de 51 ítems. Los resultados obtenidos, indicaron el poco interés por parte de las empresas mixtas para determinar las brechas existentes entre el desempeño actual propio y el de los competidores líderes. De igual manera, se evidenció la poca inversión en la incorporación de nuevas tecnologías al sector de estudio, así como fallas en un plan de formación del personal, aunado a debilidades para impulsar las alianzas estratégicas con las fuentes externas. Posteriormente, se llevó a cabo un benchmarking de tipo competitivo entre tres empresas mixtas seleccionadas, arrojando una brecha de un 59\% entre la empresa mixta $\mathrm{A}$, en comparación con las empresas mixtas A y B. Finalmente, se llevó a cabo el plan estratégico mediante la técnica del cuadro de mando integral, donde se establecieron diez objetivos estratégicos, formulados para los siete proyectos generados en la investigación, orientados a consolidar las estrategias de innovación tecnológica en el sector crudos pesados y extrapesados apoyados en la herramienta del benchmarking.
\end{abstract}

Palabras Clave: Benchmarking, Estrategia de Innovación Tecnológica, Plan estratégico, Cuadro de Mando Integral, Crudos Pesados y Extrapesados

\section{BENCHMARKING SYSTEM AS A TECHNOLOGICAL INNOVATION STRATEGY IN THE HEAVY AND EXTRAHEAVED CRUDE SECTOR}

\begin{abstract}
The general objective of the work presented was to propose a strategic plan based on benchmarking as a technological innovation strategy for the heavy and extra-heavy crude sector. The research was explanatory, field, with a feasible project modality, with a non-experimental and descriptive transectional design, the sample corresponded to six mixed companies of the heavy and extra-heavy crude sector, selected by non-probabilistic sampling based on criteria of selection. The research was developed through the survey technique, with a questionnaire of 51 items. The results obtained indicated the little interest on the part of joint ventures to determine
\end{abstract}


the existing gaps between their own current performance and that of leading competitors. Similarly, little investment in the incorporation of new technologies into the study sector was evidenced, as well as failures in a staff training plan, coupled with weaknesses to promote strategic alliances with external sources. Subsequently, a competitive benchmarking was carried out among three selected joint ventures, yielding a gap of $59 \%$ between joint venture A, compared to joint ventures A and B. Finally, the Strategic plan using the balanced scorecard technique, where ten strategic objectives were established, formulated for the seven projects generated in the research, aimed at consolidating technological innovation strategies in the heavy and extra-heavy crude sector supported in the benchmarking tool.

Keywords: Benchmarking, Technological Innovation Strategy, Strategic Plan, Balanced Scorecard, Heavy and Extra Heavy Crudes

\section{Introducción}

El contexto actual de la mejora de la competitividad empresarial, tiene lugar mediante la comparación entre empresas (Benchmarking), lo cual permite identificar las mejores prácticas y los mejores productos, procesos y servicios de las empresas líderes en el mercado, se evalúa la brecha entre las empresas en estudio con base en estos parámetros, es decir, mediante indicadores de competitividad, con el propósito de mejorar las estrategias en sus procesos innovadores, y alcanzar el conocimiento tecnológico que permita desarrollar ventajas competitivas a las empresas para lograr mantener su posición en el mercado.

En este sentido, son relevantes dos conceptos: benchmarking y estrategia de innovación tecnológica. En primera instancia, el benchmarking según Spendolini (2005), citado por Marciniak (2017), es un proceso sistemático y continuado con el propósito de evaluar y comparar los productos, servicios y procesos de trabajo de las organizaciones las cuales se reconocen como representantes de las mejores prácticas, con el propósito de realizar progresos organizacionales.

Asimismo, de acuerdo con CPCE (2009) citado por Bocángel \& Bocángel (2018), el benchmarking es un proceso donde se mide de manera continua y sistemática, a través de la comparación permanentemente de los procesos empresariales de una organización con respecto a los procesos llevado acabo por los líderes, con el propósito de obtener información que permita ayudar a la organización a desarrollar acciones que mejoren su desempeño empresarial.

Ahora bien, para llevar a cabo la técnica del benchmarking, es fundamental aplicar las etapas de planificación, análisis, integración y acción, con el propósito de determinar cual proceso, producto o servicio requiere cambios y cuáles son las empresas candidatas consideradas los mejores en sus prácticas, a fin de compararse; logrando de esta manera mejorar la competitividad de la empresa. En segunda instancia, las estrategias de innovación tecnológica, se articulan con los factores y fuentes que inciden en el esfuerzo innovador de las empresas.

Así, a través de la innovación tecnológica, según Tejada et al.(2019), se pueden incorporar nuevas ideas en los procesos, productos, métodos y conocimiento, lo cual representa un beneficio tanto para el mercado como para la sociedad; sin embargo, de acuerdo con los autores, esto dependerá de la forma como se implemente y del impacto que produzca en el mercado.

IPSA SCIENTIA - Vol. 5 No $1-2020$ 
Por otro lado, de acuerdo con la Organización de Países Exportadores de Petróleo OPEP (2020), es conocido que Venezuela posee las mayores reservas de crudos en el mundo, con aproximadamente $302 \mathrm{MMMB}$. En ese sentido, las empresas mixtas del sector crudo pesado y extrapesado, tienen la responsabilidad de incrementar la producción y para ello requieren entre otras cosas, aplicar nuevas tecnología, disminuir los costos de producción, evaluar las mejores prácticas de otras empresas, impulsar alianzas estratégicas con clientes, proveedores, universidades y empresas competidoras. Sin embargo, de acuerdo a la situación actual que atraviesa el país, los indicadores de gestión muestran una realidad compleja, donde se evidencia la falta de estrategias de innovación tecnológica en este sector.

La planificación estratégica, basada en el cuadro de mando integral, se constituye en una herramienta de gran utilidad para responder a las nuevas coyunturas. En este sentido, entre los resultados emanados del análisis del presente trabajo de investigación se encuentra una propuesta de un plan estratégico integral basado en la herramienta benchmarking como estrategia de innovación tecnológica a ser aplicado a las empresas mixtas del crudos pesados y extrapesados.

\section{Materiales y Métodos}

La presente investigación fue de tipo explicativa, de campo, basado en Hernández et al. (2014), con modalidad proyecto factible, con un diseño no experimental y transeccional descriptivo, la muestra correspondió a seis (06) empresas mixtas del sector crudos pesados y extrapesados, seleccionadas mediante muestreo no probabilístico o intencional basado en criterios de selección, estos son:

1) De las seis (06) empresas mixtas seleccionadas como muestra, cuatro (04) de ellas poseen las mayores reservas de crudo pesado y extrapesados ubicadas en la Faja Petrolífera del Orinoco, Venezuela.

2) Las seis (06) empresas mixtas pertenecen al mismo segmento de estudio, es decir, al sector recuperación mejorada de crudos pesados y extrapesados, esto basado en lo señalo por Porter (1995), citado por Batista (2012).

3) Son empresas petroleras internacionales de trayectoria, con las cuales Petróleos de Venezuela, S.A. ha llevado a cabo la conformación de las diferentes empresas mixtas del sector crudos pesados y extrapesados, esto basado en los principios del benchmarking (comparación con los mejores), dichas empresas son: Chevron (Estados Unidos), Total (Francia), Statoil (Noruega), Rosneft (Rusia).

4) Se consideraron los indicadores más relevantes de acuerdo al informe del Comisario de PDVSA (2017), tales como: operacionales de producción, pozos activos y actividades con taladros, entre otros, en los cuales cinco (05) empresas ocuparon los primeros lugares en dichos indicadores.

IPSA SCIENTIA - Vol. $5 N^{\circ} 1-2020$ 
5) De las seis (06) empresas mixtas seleccionadas, cuatro (04) de ellas operan plantas de mejoramientos de crudos extrapesados, lo que evidencia su potencial capacidad de producción, PDVSA (2017).

6) Fue seleccionada una empresa $100 \%$ propiedad del estado venezolano (CVP), es decir, es una empresa mixta con esfuerzo propio, dicha empresa será objeto de comparación con el resto de las empresas, aplicando la herramienta benchmarking.

Partiendo de esta premisa, es importante señalar que la presidencia de cada una de estas empresas seleccionadas, así como sus respectivas gerencias generales, son piezas fundamentales para obtener la información. En este sentido, el investigador seleccionó intencionalmente los sujetos, a través de las cuales se obtuvo la información requerida en el presente trabajo. Los sujetos seleccionados fueron, los seis (06) presidentes de cada una de las empresas mixtas de crudos pesados y extrapesados, junto los seis (06) gerentes generales, de cada empresa mixta. Tal como se muestra en el cuadro 1 :

Cuadro 1. Características de la población

\begin{tabular}{|c|c|c|c|}
\hline \multicolumn{3}{|c|}{ EMPRESAS MIXTAS SELECCIONADAS - INFORMANTES } \\
\hline \multirow{2}{*}{$\mathrm{N}^{\circ}$} & \multirow{2}{*}{ Empresa mixta } & \multicolumn{2}{|c|}{ Informantes } \\
\cline { 3 - 4 } & & Presidente & Gerente General \\
\hline 1 & Empresa mixta A & 1 & 1 \\
\hline 2 & Empresa mixta B & 1 & 1 \\
\hline 3 & Empresa mixta C & 1 & 1 \\
\hline 4 & Empresa mixta D & 1 & 1 \\
\hline 5 & Empresa mixta E & 1 & 6 \\
\hline 6 & Empresa mixta F & 6 & 1 \\
\hline \multicolumn{2}{|c|}{} \\
\hline
\end{tabular}

Fuente: Elaboración propia (2020)

La investigación se desarrolló mediante la técnica de encuesta, para la elaboración del instrumento de recolección de datos, fue considerado el marco teórico. Partiendo de esta base fue diseñado el cuestionario denominado BENCH-001, aplicado en el presente trabajo, el mismo abordó (51) preguntas cerradas derivadas de los 17 indicadores que agrupan las dos variables objeto de estudio: benchmarking y estrategia de innovación tecnológica y cuyas dimensiones corresponden a las etapas y tipos de benchmarking, así como los factores y fuentes de la estrategia de innovación tecnológica.

Cabe destacar, que en dicho cuestionario se aplicó la escala Likert, los cuales corresponden a: siempre, casi siempre, algunas veces, casi nunca y nunca. Asimismo, fue validado mediante juicio de expertos, con una alta confiabilidad $(0,80)$, en el coeficiente Alfa Cronbach. Por otra parte, la técnica de análisis de datos utilizada en el presente trabajo , correspondió a la estadística descriptiva, específicamente la distribución de frecuencias y las medidas de tendencia de central (media), para lo cual se utilizaron como herramientas de apoyo los programas Microsoft Excel, y el Statistical Package for the Social Sciences (SPSS).

IPSA SCIENTIA - Vol. $5 N^{\circ} 1-2020$ 
Sistema de benchmarking como estrategia de innovación tecnológica en el sector

crudos pesados y extrapesados

Con respecto a la interpretación de los datos, se estableció un baremo de interpretación; con el propósito de efectuar el proceso de confrontación de los resultados obtenidos durante el proceso de recolección de datos, tanto para la media aritmética como para la desviación estándar.

Finalmente, el procedimiento desarrollado en el presente trabajo, considero las etapas descritas a continuación:

1. Revisión de la literatura con el objeto de identificar las variables objeto de estudio.

2. Revisión de los antecedentes y documentación respecto a las variables objeto de estudio.

3. Desarrollo del planteamiento del problema relacionado con las variables de investigación.

4. Definición de los objetivos de la investigación.

5. Desarrollo de las bases teóricas, en función de las variables objeto de estudio.

6. Definición de la muestra poblacional según la información recolectada.

7. Definición del tipo de investigación y el diseño correspondiente.

8. Diseño y validación del instrumento de recolección de la información relacionada con la investigación.

9. Aplicación del cuestionario a la prueba piloto, con el objeto de calcular su confiabilidad.

10. Aplicación de los instrumentos a la población objeto de estudio de la investigación.

11. Tabulación y aplicación de técnicas estadísticas a los datos obtenidos de la aplicación de los instrumentos de recolección de información.

12. Análisis y discusión de los resultados obtenidos en la investigación.

13. Aplicación del benchmarking de tipo competitivo entre las empresas mixtas objeto de estudio.

14. Diseño de un plan estratégico basado en el benchmarking como estrategia de innovación tecnológica para la extracción de crudos pesados.

15. Elaboración de las conclusiones respecto a las variables de la investigación.

\section{Resultados y discusión}

Luego de recopilados los datos del cuestionario, aplicado a los seis (06) presidentes y seis (06) gerentes generales de las seis (06) empresas mixtas del sector crudos pesados y extrapesados, se IPSA SCIENTIA - Vol. 5 No $^{\circ}-2020$ 
procedió a realizar el análisis de los datos mediante los procesos de codificación, procesamiento de datos y presentación de los resultados. Con respecto a las etapas del benchmarking basado en el modelo de Camp, para la extracción de crudos pesados y extrapesados estos fueron los resultados mostrados en la tabla 1 :

Tabla 1. Etapas del benchmarking

\begin{tabular}{|c|c|c|c|c|c|c|c|c|c|c|c|c|c|c|c|c|}
\hline \multirow[t]{2}{*}{ Indicadores } & \multicolumn{2}{|c|}{ Nunca } & \multicolumn{2}{|c|}{$\begin{array}{c}\text { Casi } \\
\text { nunca }\end{array}$} & \multicolumn{2}{|c|}{$\begin{array}{c}\text { Algunas } \\
\text { veces }\end{array}$} & \multicolumn{2}{|c|}{$\begin{array}{c}\text { Casi } \\
\text { siempre }\end{array}$} & \multicolumn{2}{|c|}{ Siempre } & \multicolumn{2}{|c|}{ Total } & \multirow[t]{2}{*}{$\bar{X}$} & & \multirow{2}{*}{\multicolumn{2}{|c|}{$\Sigma$}} \\
\hline & $\mathrm{Fa}$ & $\%$ & $\mathrm{Fa}$ & $\%$ & $\mathrm{Fa}$ & $\%$ & $\mathrm{Fa}$ & $\%$ & $\mathrm{Fa}$ & $\%$ & $\mathrm{Fa}$ & $\%$ & & & & \\
\hline Planificación & 0 & 0 & 1 & 3 & 6 & 17 & 21 & 58 & 8 & 22 & 36 & 100 & 4,00 & $P$ & 0,61 & $\mathrm{BD}$ \\
\hline Análisis & 0 & 0 & 1 & 3 & 21 & 58 & 12 & 33 & 2 & 6 & 36 & 100 & 3,42 & $\mathrm{P}$ & 0,65 & $\mathrm{BD}$ \\
\hline Integración & 0 & 0 & 1 & 3 & 15 & 42 & 11 & 31 & 9 & 25 & 36 & 100 & 3,78 & $P$ & 0,56 & $\mathrm{BD}$ \\
\hline Acción & 0 & 0 & 0 & 0 & 11 & 31 & 15 & 42 & 10 & 28 & 36 & 100 & 3,97 & $P$ & 0,77 & DI \\
\hline
\end{tabular}

Fuente: Elaboración propia (2020)

De la tabla anterior, se observa, que dichas etapas se encuentran $100 \%$ presentes, es relevante destacar que, si bien, se registra presencia de las etapas del benchmarking, la etapa análisis registra un menor valor frente a las otras etapas, lo que pone de manifiesto el poco interés para determinar las diferencias existentes entre el desempeño actual propio y el del los competidores lideres, de acuerdo con Hernández y Cano (2017).

En lo referente al tipo de benchmarking aplicado en el contexto de las empresas expertas en crudos pesados y extrapesados, se presentan los siguientes resultados mostrados en la tabla 2:

Tabla 2. Tipos de benchmarking

\begin{tabular}{|c|c|c|c|c|c|c|c|c|c|c|c|c|c|c|c|c|}
\hline \multirow{2}{*}{$\begin{array}{l}\text { Indicadores/ } \\
\text { Ítems }\end{array}$} & \multicolumn{2}{|c|}{ Nunca } & \multicolumn{2}{|c|}{$\begin{array}{c}\text { Casi } \\
\text { nunca }\end{array}$} & \multicolumn{2}{|c|}{$\begin{array}{l}\text { Algunas } \\
\text { veces }\end{array}$} & \multicolumn{2}{|c|}{$\begin{array}{c}\text { Casi } \\
\text { siempre }\end{array}$} & \multicolumn{2}{|c|}{ Siempre } & \multicolumn{2}{|c|}{ Total } & \multirow{2}{*}{\multicolumn{2}{|c|}{$\bar{X}$}} & \multirow{2}{*}{\multicolumn{2}{|c|}{$\Sigma$}} \\
\hline & $\mathrm{Fa}$ & $\%$ & $\mathrm{Fa}$ & $\%$ & $\mathrm{Fa}$ & $\%$ & $\mathrm{Fa}$ & $\%$ & $\mathrm{Fa}$ & $\%$ & $\mathrm{Fa}$ & $\%$ & & & & \\
\hline Interno & 0 & 0 & 0 & 0 & 12 & 33 & 21 & 58 & 3 & 8 & 36 & 100 & 3,75 & $\mathrm{P}$ & 0,57 & $\mathrm{BD}$ \\
\hline Competitivo & 0 & 0 & 0 & 0 & 11 & 31 & 19 & 53 & 6 & 17 & 36 & 100 & 3,86 & $\mathrm{P}$ & 0,61 & $\mathrm{BD}$ \\
\hline Genérico & 0 & 0 & 0 & 0 & 15 & 42 & 21 & 58 & 0 & 0 & 36 & 100 & 3,58 & $\mathrm{P}$ & 0,47 & $\mathrm{BD}$ \\
\hline Funcional & 0 & 0 & 2 & 6 & 18 & 50 & 16 & 44 & 0 & 0 & 36 & 100 & 3,39 & $P$ & 0,52 & $\mathrm{BD}$ \\
\hline
\end{tabular}

Fuente: Elaboración propia (2020) 
En los resultados se observa presencia de los cuatros tipos de benchmarking; sin embargo, a pesar de que se registra presencia de estos indicadores, se evidencia la poca iniciativa por parte de la empresa mixta de llevar a cabo un benchmarking, bien sea por la limitante al acceso de información, equipos y métodos de la competencia, o por sanciones impuestas a empresas de otros países, lo cual no permite llevar a cabo las alianzas estratégicas.

Esto coincide con lo planteado por los autores Hernández y Cano (2017), al referirse a que es complejo que las empresas acepten medirse con las mejores en su sector; sin embargo, dicha comparación es necesario cuando se desea conocer las estrategias en servicios, productos y procesos, que han empleado estas empresas para llegar al punto donde se encuentran. Con respecto a los factores que influyen en las estrategias de innovación tecnológica para la extracción de crudos pesados y extrapesados, se presentan los resultados de la tabla 3.

Se observa presencia del factor tamaño de la empresa, considerando las empresas mixtas, la posibilidad de que las empresas grandes tienen mayor oportunidad de ser más innovadoras, principalmente por contar con los recursos y personal cualificado. Por su parte, los factores nivel de formación, secreto industrial, redes de cooperación y obstáculos a la innovación reportan mediana presencia en las empresas analizadas

En general, se evidencia deficiencia asociadas a un plan de formación del personal que permita fortalecer el conocimiento en la empresa, contrario a lo señalado por Ortega (2019) quien asegura que la conformación de equipos de trabajo con personal de un alto nivel de educación, técnicamente cualificados y experimentados en diversas especialidades, es un factor determinante en el proceso innovador.

Tabla 3. Factores que influyen en las estrategias de innovación tecnológica

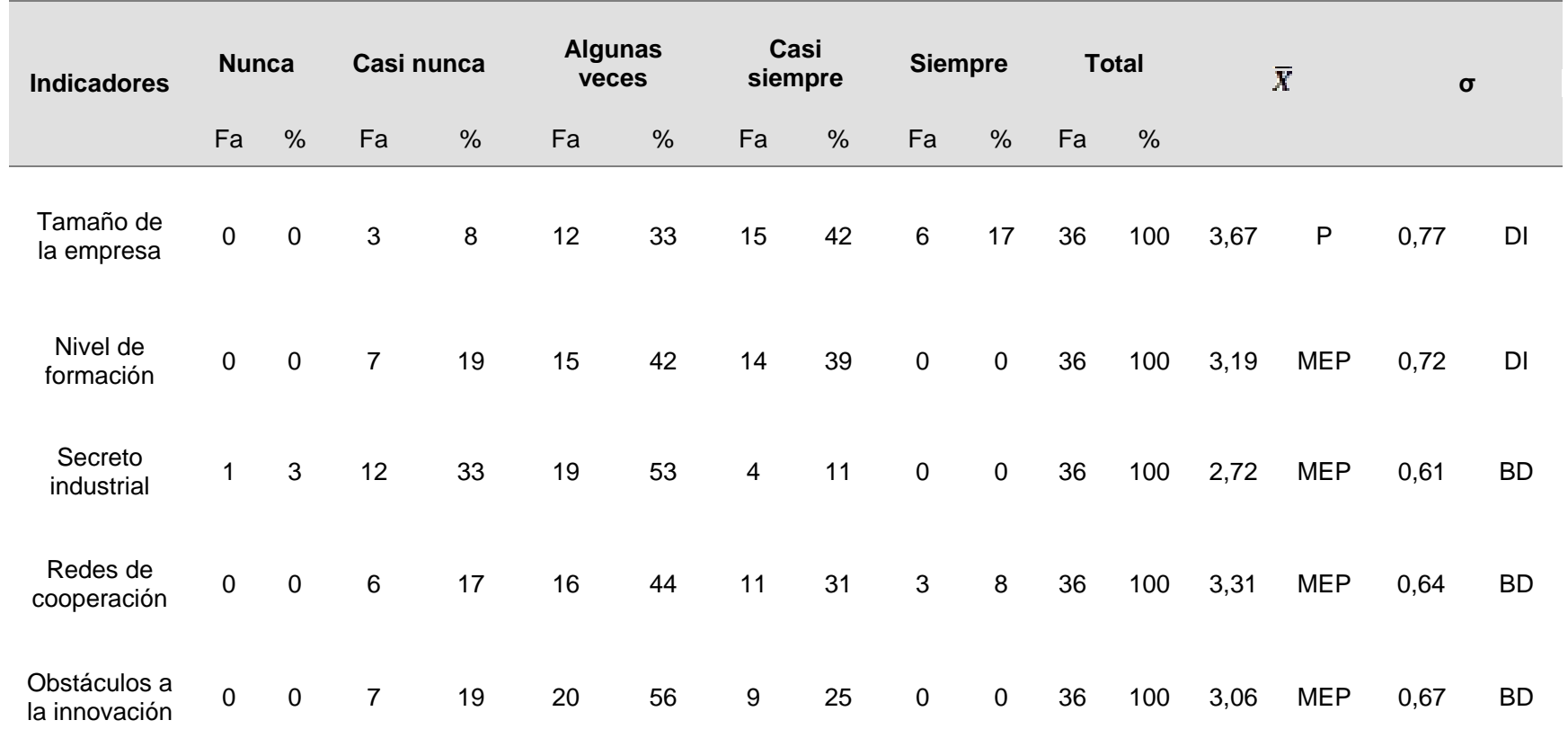

Fuente: Elaboración propia (2020)

IPSA SCIENTIA - Vol. 5 NN$^{\circ} 1-2020$ 
De igual manera, se observa bajos niveles de apropiación de la innovación., así como poca disponibilidad por parte de la empresa mixta de llevar acabo alianzas estratégicas con otros agentes industriales y agentes tecnológicos. En lo referente a las fuentes de la estrategia de innovación tecnológica empleadas en el contexto de la extracción de crudos pesados y extrapesados, la tabla 4 presenta los siguientes resultados:

Tabla 4. Fuentes de la estrategia de innovación tecnológica

\begin{tabular}{|c|c|c|c|c|c|c|c|c|c|c|c|c|c|c|c|c|}
\hline \multirow{2}{*}{$\begin{array}{l}\text { Indicadores/ } \\
\text { Ítems }\end{array}$} & \multicolumn{2}{|c|}{ Nunca } & \multicolumn{2}{|c|}{$\begin{array}{c}\text { Casi } \\
\text { nunca }\end{array}$} & \multicolumn{2}{|c|}{$\begin{array}{l}\text { Algunas } \\
\text { veces }\end{array}$} & \multicolumn{2}{|c|}{$\begin{array}{c}\text { Casi } \\
\text { siempre }\end{array}$} & \multicolumn{2}{|c|}{ Siempre } & \multicolumn{2}{|c|}{ Total } & \multirow{2}{*}{\multicolumn{2}{|c|}{$\bar{x}$}} & \multirow{2}{*}{\multicolumn{2}{|c|}{$\sigma$}} \\
\hline & $\mathrm{Fa}$ & $\%$ & $\mathrm{Fa}$ & $\%$ & $\mathrm{Fa}$ & $\%$ & $\mathrm{Fa}$ & $\%$ & $\mathrm{Fa}$ & $\%$ & $\mathrm{Fa}$ & $\%$ & & & & \\
\hline Actividades I+D & 0 & 0 & 5 & 14 & 16 & 44 & 15 & 42 & 0 & 0 & 36 & 100 & 3,28 & MEP & 0,70 & $\mathrm{DI}$ \\
\hline $\begin{array}{c}\text { Fuentes } \\
\text { comerciales }\end{array}$ & 0 & 0 & 0 & 0 & 3 & 8 & 13 & 36 & 20 & 56 & 36 & 100 & 4,47 & MP & 0,57 & $\mathrm{BD}$ \\
\hline $\begin{array}{c}\text { Fuentes } \\
\text { institucionales }\end{array}$ & 0 & 0 & 6 & 17 & 24 & 67 & 5 & 14 & 1 & 3 & 36 & 100 & 3,03 & MEP & 0,59 & $\mathrm{BD}$ \\
\hline $\begin{array}{l}\text { Fuentes de } \\
\text { información } \\
\text { general }\end{array}$ & 0 & 0 & 3 & 8 & 21 & 58 & 12 & 33 & 0 & 0 & 36 & 100 & 3,25 & MEP & 0,58 & $\mathrm{BD}$ \\
\hline
\end{tabular}

Fuente: Elaboración propia (2020)

De la tabla anterior, se observa que las fuentes comerciales analizadas se encuentran muy presentes, esto debido a la importancia de las relaciones con los agentes externos como clientes, proveedores, competidores y laboratorios comerciales, dichos agentes permiten a las empresas adquirir conocimiento, que de acuerdo con Aparicio (2009), citado por Linares \& Geizzelez (2016), es una capacidad que origina valor mientras se mantiene dinámico, es decir, se transforma produciendo como consecuencia, el uso de la inferencia y la capacidad de razonamiento, además supone la existencia de relaciones a través de las redes con otras entidades. Asimismo, mediante estas fuentes comerciales se obtiene especialización tecnológica, lograr reducir los costos de los componentes, así como conocer las tendencias del mercado, entre otros.

Por otra parte, se evidencia debilidades en las fuentes institucionales para impulsar alianzas con las universidades, así como poco acceso de las empresas al conocimiento mediante revistas, base de datos de patentes y conferencias. De igual manera, las empresas mixtas presenta poca capacidad para generar el conocimiento interno (actividades I+D).

Luego de analizados los datos obtenidos de aplicar la estadística descriptiva, se procedió a aplicar la herramienta benchmarking definida por Benavides et al. (2018), como un método y proceso de gestión estratégica, el cual consiste en analizar empresas, organizaciones y/o sectores, consideradas como las mejores para aprender de ellas (os) por medio de una comparación. Por su parte, para Spendolini (2005), citado por Marciniak (2017), es un proceso sistemático y continuado con el fin de evaluar y comparar los productos, servicios y los procesos de trabajo de las organizaciones reconocidas como las mejores, con el propósito de realizar progresos organizacionales.

IPSA SCIENTIA - Vol. 5 No $^{\circ}-2020$ 
Del mismo modo, Mencias \& Rodríguez (2017), señalan que el benchmarking es una técnica que permite comparar, analizar y evaluar los procesos, bienes, servicios o estrategias, con aquellas empresas tanto interna o externa la finalidad de establecer diferencias 0 semejanzas que permitan generar y aplicar estrategias muy propias a su realidad para mejorar sus ventajas competitivas.

Cabe destacar que el tipo de benchmarking aplicado fue de tipo competitivo, basado Hernández y Cano (2017), quienes señalan que en este tipo de benchmarking, se produce la comparación de los estándares de una organización, con los de otras empresas comptidoras. Asimismo, de acuerdo con los autores, este tipo de benchmarking resulta el más complicado, debido a que los competidores reservan sus ventajas competitivas para sí mismos.

De este modo, en la herramienta benchmarking se debe seleccionar la empresa objeto de comparación, en este caso fue la empresa mixta C, comparada con las dos empresas mixtas más sobresalientes en la recopilación de datos (benchmark). Luego de realizada la comparación entre las empresas mixtas A, B y C, se pudo observar en líneas generales que existe una brecha de casi el 59\% por debajo de la empresa mixta C con respecto a las empresas mixtas A y B en de los indicadores, principalmente en la planificación, análisis, integración, acción, benchmarking funcional, secreto industrial, redes de cooperación, actividades de I+D y fuentes comerciales. Tal como se muestra, en la figura 1 :

Figura 1. Brechas entre las empresas mixtas A, B y C del sector crudos pesados y extrapesados

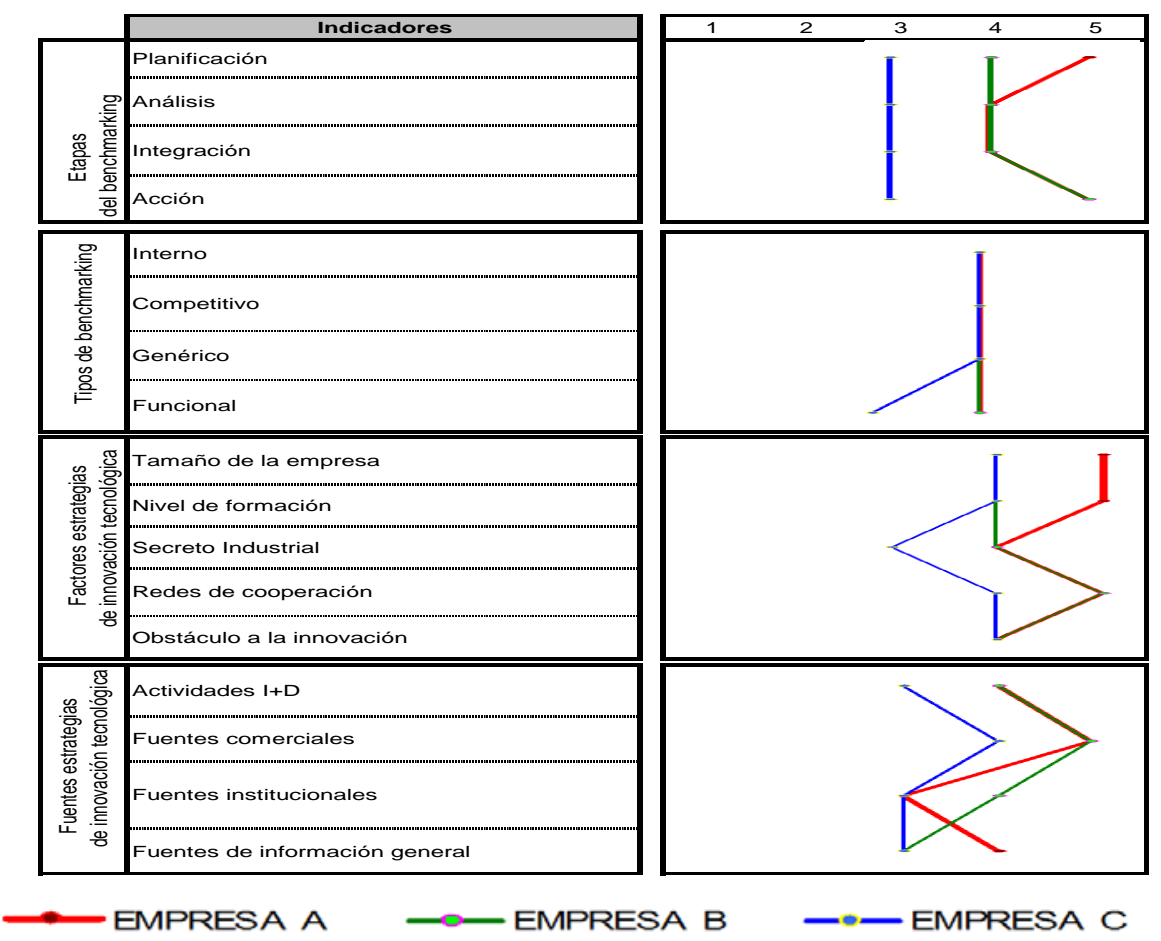

Fuente: Elaboración propia (2020). 
Asimismo, se procedió a realizar el diseño del plan estratégico, el cual según Abascal (2004), citado por Abad (2019), determina el propósito de la organización y describe un conjunto de metas y objetivos, generando una base para la toma de decisiones que permita crear las oportunidades y transformar las amenazas en oportunidades.Por otro lado, la estrategia de innovación tecnológica, según Fernández (2005) citado por Aponte (2015), debe expresarse dentro del marco conceptual dado por la visión de la organización, la cual condiciona los objetivos tecnológicos. En este sentido, dicha visión desde el punto tecnológico de las empresas mixtas, debe ser lo suficientemente flexible como para permitir modificarse a largo plazo dependiendo del cambio de los objetivos, debido a que estos son impactados por los cambios del entorno. Por su parte, según Correa (2012), citado por Paternina et al. (2019), la estrategia tecnológica, es un proceso que involucra la adquisición tecnológica, en el cual la innovación requiere una demanda de recursos e insumos con el propósito de crear oportunidades para desarrollar otros productos y procesos.

En este sentido, se llevo cabo el plan estratégico para el sector crudos pesados y extrapesados, que permita a la empresa mixta C, además de acortar dichas brechas, alcanzar las mejores prácticas, para ello, se utilizó la metodología propuesta por el autor David (2013), citado por Pérez (2019), en primer lugar se realizó la formulación de estrategias, donde se revaloró la misión y visión, luego se procedió a realizar el análisis del entorno tanto externo (oportunidadesamenazas) como interno (fortalezas-debilidades); y finalmente para el diseño de estrategias se establecieron tres fases: fase 1- insumos: matriz FODA, matriz de evaluación de factores externos e internos, fase 2- adecuación: matriz estratégica, jerarquización de estrategias y fase 3decisión: matriz cuantitativa, objetivos estratégicos. Con respecto, al cuadro de mando integral, según Abad (2019), este provee una estructura para centrarse en los indicadores de cada proceso crítico, como: plan de negocio, recursos, estrategias, aprendizaje, y clientes. Sobre lo anterior, esta técnica del cuadro de mando integral permitió medir las actividades de la empresa en términos de su visión y estrategia definidos por el plan estratégico. Según Baptista (2012), dicho modelo de medición del desempeño fue desarrollado por Kaplan y Norton (2000).A este respecto, este modelo involucra indicadores tanto financieros como no financieros, basados en cuatro áreas fundamentales de la organización: (1) finanzas, (2) clientes, (3) aprendizaje y (4) procesos internos. A continuación, se muestra en la figura 2, la propuesta de como se desarrollo el plan estratégico basado en las variables objeto de estudio.

Figura 2: Propuesta para el diseño del plan estratégico.

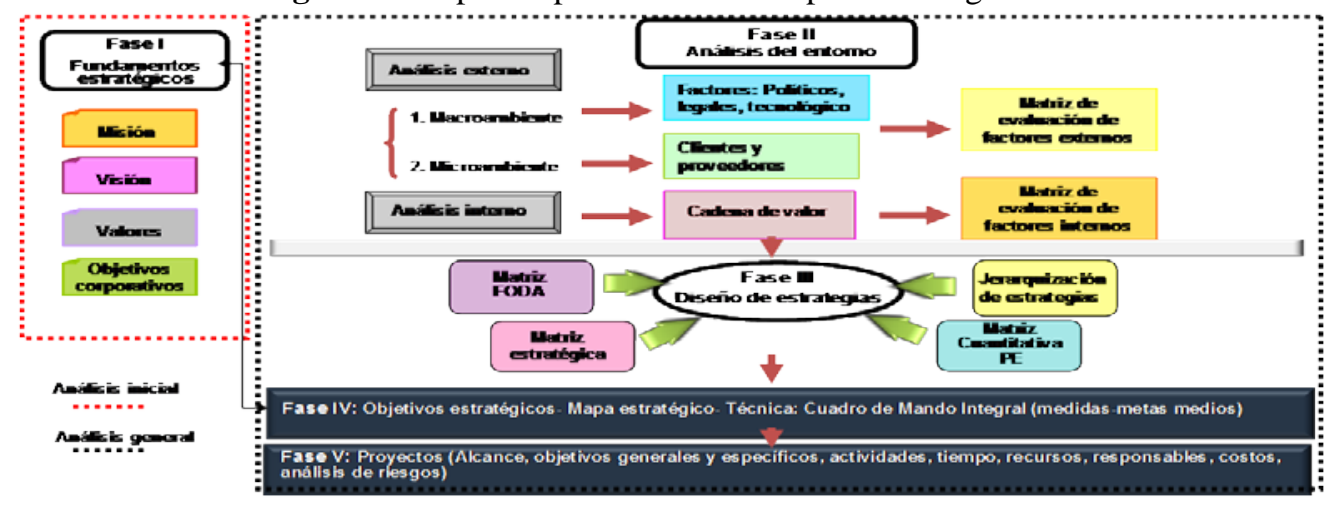

Fuente: Elaboración propia (2020)

IPSA SCIENTIA - Vol. 5 No $^{\circ} 1-2020$ 
Con el fin de proporcionar a la empresa mixta $\mathrm{C}$ del sector crudos pesados y extrapesados las herramientas de control de la gestión administrativa que le permitan dar a conocer su misión, visión e implementar sus estrategias y objetivos de forma eficiente, fue elaborado un plan estratégico basado en el benchmarking como estrategia de innovación tecnológica aplicando el cuadro de mando integral que permitirá establecer indicadores de gestión basados en las cuatro perspectivas, clientes, proceso internos, aprendizaje y financiera, determinando las mediciones, metas y medios para cada una. Mediante la aplicación de instrumentos de recolección de datos obtenidos de los presidentes y gerentes generales de las empresas mixtas se determinaron los siguientes problemas a analizar:

1. Decreciente producción de crudos

2. Bajo niveles en los indicadores de competitividad

3. Limitado desarrollo de planes de formación del personal

4. Limitada alianzas estratégicas con agentes externos (clientes, proveedores, competidores y universidades)

5. Poca inversión en nuevas tecnologías de recuperación mejorada de crudos pesados y extrapesados

\section{a) Fundamentos estratégicos}

Los fundamentos estratégicos guiarán en el mediano y largo plazo el funcionamiento de la empresa mixta del sector crudos pesados y extrapesados; en tal sentido, la fase de orientación en la planeación estratégica estuvo conformada por la revaloración de la misión, visión de la empresa mixta $\mathrm{C}$, que forman parte de la cultura organizacional y están vinculados a los objetivos corporativos de dicha empresa.

Misión

Desarrollar actividades estratégicas de recuperación mejorada de hidrocarburos de excelente calidad dentro de un mercado global, promoviendo íntegramente las relaciones con nuestro personal, clientes, proveedores y colaboradores, alcanzando el conocimiento tecnológico, crecimiento, rentabilidad y solidez con responsabilidad social, alineados con el Plan de la Patria para contribuir al desarrollo energético de Venezuela.

Visión

Ser una empresa energética modelo, basada en la innovación, la eficiencia y el respeto, con el propósito de crear valor de manera sostenible en el desempeño en el sector petrolero para el progreso de la sociedad venezolana.

\section{b) Análisis del entorno}

La formulación estratégica inicia con el análisis del entorno conformado por un análisis externo (macroambiente y microambiente) y un análisis interno (cadena de valor), de acuerdo con Marcillo y Garzón (2014). Atendiendo a lo anteriormente descrito y partiendo del diseño del plan

IPSA SCIENTIA - Vol. 5 No $^{\circ}-2020$ 
estratégico para la empresa mixta del sector crudo pesado y extrapesado, este análisis del entorno trata de una investigación profunda en determinado momento al entorno en el cual se desenvuelve la empresa mixta del sector crudos pesado y extrapesado, de tal manera que sirva de base para seguir el proceso de planificación estratégica.

\section{Análisis externo}

Este análisis trata de las condiciones generales del entorno llamado macroambiente, dentro de las cuales se encuentran los factores, económicos, políticos, sociales, tecnológicos, entre otros, que afectan a la vida de la empresa, así como también, las condiciones específicas del mercado, los clientes y proveedores (microambiente). Para el caso de la empresa mixta del sector crudo pesado y extrapesado, el macroambiente y microambiente donde se analizaron los factores externos antes mencionados, estudiados en profundidad sobre los aspectos de las variables benchmarking y estrategia de innovación tecnológica, así como otros elementos complementarios, permitieron identificar diez (10) factores clave que ocurren en el ambiente externo, los cuales se analizaron a fin de determinar las oportunidades y amenazas del sector de estudio

Matriz de evaluación de factores externos (EFE)

Una vez identificadas las oportunidades y amenazas, se procedió a la elaboración de la Matriz de evaluación de factores externos (EFE), en la cual se organizaron los factores externos distribuidos en cinco (5) factores para las oportunidades y cinco (5) factores para las amenazas. El puntaje obtenido de la matriz EFE fue de 2,70, este resultado indica que está ligeramente por encima de la media teórica de 2,5, siendo las oportunidades aprovechadas medianamente, por parte de la empresa mixta A.

\section{Análisis interno:}

El análisis interno, permite obtener información sobre las áreas funcionales (cadena de valor) a fin de identificar las fortalezas y debilidades inherentes con la posición en cuanto a recursos, así como capacidades que permiten determinar si posee fuerza competitiva suficientemente robusta frente a los rivales en el mercado. Para el caso de la empresa mixta del sector crudos pesados y extrapesados, se analizaron los factores internos clave que conforman su cadena de valor, desde el proceso de exploración, perforación, producción, mejoramiento, refinación, comercialización hasta las relaciones existentes con clientes y proveedores. Los cuales permitieron identificar 10 factores debilidades del sector de estudio

Matriz de evaluación de factores internos (EFI)

De igual manera para la matriz EFI, se determinaron los factores de éxito internos que incluyen fortalezas como debilidades, con un peso de $50 \%$ a tanto a las fortalezas como a las debilidades se pudo observar el puntaje obtenido de la matriz de evaluaciones de factores internos (EFI) fue de 2,35, este resultado indica para el caso de la empresa mixta del sector crudos pesados y

IPSA SCIENTIA - Vol. $5 N^{\circ} 1-2020$ 
extrapesados está por debajo de la media teórica de 2,5, lo cual representa un posicionamiento interno muy débil.

\section{c) Diseño de Estrategias}

Para llevar a cabo el diseño de estrategias en el plan estratégico propuesto, inicialmente se debió considerar los resultados obtenidos del análisis estratégico del entorno, tanto el externo como el interno, del cual se obtuvo la fase de adecuación de insumos. El propósito fue realizar el análisis de matriz FODA de las matrices obtenidas del análisis estratégico del entorno para la empresa mixta, que junto a la selección de estrategias por medio de la Matriz Cuantitativa de la Planificación Estratégica (MCPE), conformaron el diseño de estrategias propuestas.

\section{Adecuación de insumos}

De acuerdo con David (2013) citado por Pérez (2019), durante esta fase del plan estratégico, se utilizaron los factores claves, tanto externos como internos, resultados del análisis estratégico de entorno detallado en las matrices EFE y EFI para analizarlos en la matriz FODA. En este sentido, la matriz FODA generó 04 tipos de estrategias combinando factores claves por cuadrante. Ver tabla 5 de la matriz estratégica.

Cuadro de Mando Integral.

Como ya se mencionó, el cuadro de mando integral es un instrumento de gestión que permite la implantación de la estrategia de la empresa de una forma eficiente, debido a que proporciona la estructura adecuada para comunicar la misión y la estrategia en objetivos e indicadores organizados en cuatro perspectivas: finanzas, clientes, procesos internos y aprendizaje. En este sentido, una vez definido el instrumento cuadro de mando integral, se procedió a establecer los objetivos estratégicos dentro de las cuatro perspectivas del CMI; es decir finanzas, clientes, procesos internos y aprendizaje.

Seguidamente fue necesario alinear las doce (12) estrategias generadas por la matriz FODA con los diez (10) objetivos estratégicos en el tiempo, para posteriormente determinar su ejecución. A continuación se muestran la alineación de las estrategias y objetivos estratégicos en la técnica del cuadro de mando integral, en el corto, mediano y largo plazo:

Objetivos estratégicos

Corto plazo (hasta 1 año):

1. Incrementar la rentabilidad de la gestión de los recursos disponibles (E11)

8. Fortalecer el nivel de formación del personal acorde a las exigencias de la empresa (E6)

9. Mejorar las condiciones laborales que permita aumentar el nivel de satisfacción del empleado (E10)

Mediano plazo (de 1 a 5 años):

IPSA SCIENTIA - Vol. 5 No $^{\circ}-2020$ 
2. Garantizar el soporte tecnológico en los procesos de la empresa, asegurando la satisfacción de los clientes (E3)

4. Impulsar alianzas estratégicas con los principales proveedores (E12)

5. Establecer alianzas estratégicas con las mejores empresas del sector (E2, E9)

Largo plazo (hasta 10 años):

3. Desarrollar las reservas de crudos pesados y extrapesados incrementando el factor de recobro en un $20 \%$ (E1)

6. Innovar la tecnología de la empresa a fin de mejorar el proceso de producción de crudos (E4, E8).

7. Fortalecer la eficiencia en las operaciones a fin de alcanzar los niveles de producción de crudos (E5)

Mapa estratégico

A partir de los objetivos estratégicos, se diseñó el mapa estratégico, alineando cada una de las perspectivas y que estas se relacionan naturalmente, ver figura 3. Asimismo, se determinaron los factores claves correspondientes a las cuatro perspectivas definidas que permitieran la consecución de los mismos, y que guarden relaciones de causa-efecto. En este sentido, se establecieron también las áreas críticas donde se encuentra los factores claves. Seguidamente se eligieron ocho (08) indicadores que guardaran una estructura y consistencia lógica a fin de encontrar la relación causa-efecto, es decir, de crear un equilibrio entre los indicadores de cada perspectiva.

Asimismo, se establecieron las metas de las cuales se logra el alcance de los objetivos fijados, para la elaboración de dichas metas se utilizó del mismo modo el Cuadro de Mando Integral como una herramienta de gestión estratégica para impulsar un cambio en la empresa. Estas metas serán alcanzadas en un horizonte de tiempo para cada indicador, proyectando ubicar a la empresa en la posición deseada. De igual manera, se establecieron los medios, es decir, las actividades, los proyectos y las acciones en las que la empresa debe enfocarse para alcanzar las metas establecidas. Esto se recoge en las tablas 6 y 7, donde se muestra el cuadro de mando integral con las metas, medidas y medios establecidos en el plan estratégico. 


\begin{tabular}{|c|c|c|}
\hline & OPORTUNIDADES & AMENAZAS \\
\hline $\begin{array}{l}\text { FACTORES } \\
\text { EXTERNOS }\end{array}$ & $\begin{array}{l}\mathbf{O}_{1} \text {.Agotamiento de las reservas convencionales } \\
\mathrm{O}_{2} \text {.Aprendizaje de las instituciones de investigación } \\
\text { para alcanzar el conocimiento tecnológico } \\
\mathrm{O}_{3 .} \text { Participación con empresas del sector para } \\
\text { mejorar las prácticas de recuperación mejorada de } \\
\text { crudos } \\
\mathbf{O}_{4} \text {. Beneficios de la experiencia y conocimientos } \\
\text { adquiridos por los proveedores y clientes } \\
\mathbf{0}_{5} \text {.Posibilidad de adquirir nuevas tecnologías para la } \\
\text { recuperación mejorada de crudos }\end{array}$ & $\begin{array}{l}\mathbf{A}_{1} \text {. Deudas contractuales con proveedores de } \\
\text { bienes y servicios } \\
\mathbf{A}_{2} \text {. Empresas del sector con políticas de desarrollo } \\
\text { y beneficios competitivos que atraen al talento } \\
\text { humano capacitado } \\
\mathbf{A}_{3} \text {. Dificultad para el acceso a la financiación } \\
\text { externa (sanciones) } \\
\mathbf{A}_{4} \text {. Disponibilidad y acceso para la tecnología } \\
\mathbf{A}_{5} \text {. Dificultad para acceder a la información de } \\
\text { empresas competidoras, a fin de establecer una } \\
\text { comparación. }\end{array}$ \\
\hline FORTALEZAS & ESTRATEGIAS FO & ESTRATEGIAS FA \\
\hline $\begin{array}{l}\text { F. Las reservas de petróleo más grande del mundo } \\
\text { F. }_{2} \text { Presencia de socios internacionales con } \\
\text { experiencia en las empresas mixtas } \\
\text { F }_{3} \text {.Procesos y experiencia que facilitan el } \\
\text { aprendizaje de otras empresas, a fin de fortalecer } \\
\text { sus operaciones } \\
\text { F }_{4} \text { Propósito de prestar un servicio de mejor calidad } \\
\text { a sus clientes } \\
\text { F }_{5} \text {.Determinación de modernizar la tecnología de la } \\
\text { empresa para optimizar los procesos }\end{array}$ & $\begin{array}{l}\mathbf{E}_{1} \text {. Desarrollar las reservas de crudos pesados y } \\
\text { extrapesados atendiendo al agotamiento de las } \\
\text { reservas de crudos convencionales en el mundo } \\
\left(F_{1}, \mathbf{O}_{1}\right) \\
\mathbf{E}_{2} \text {. Establecer sólidas alianzas estratégicas con } \\
\text { empresas expertas en el sector petrolero, a fin de } \\
\text { aprender de sus procesos }\left(F_{2}, F_{3}, O_{3}\right) \\
E_{3} \text {. Consolidar las relaciones con los clientes que } \\
\text { conforman la cadena de valor de la empresa }\left(F_{4}, \mathbf{O}_{4}\right) \\
\mathbf{E}_{4} \text {. Aplicar nuevas tecnologías que contribuyan a } \\
\text { mejorar los procesos internos }\left(F_{5}, 0_{5}\right)\end{array}$ & $\begin{array}{l}\text { E. Implementar nuevas tecnologías de vanguardia } \\
\text { que permitan aumentar el factor de recobro de } \\
\text { crudos }\left(\mathbf{F}_{1}, \mathbf{F}_{5}, \mathbf{A}_{4}\right) \\
\mathbf{E}_{9} \text {. Suscribir alianzas de cooperación con } \\
\text { empresas de la competencia que coadyuven al } \\
\text { logro de los objetivos }\left(\mathbf{F}_{2}, \mathbf{F}_{3}, \mathbf{A}_{5}\right)\end{array}$ \\
\hline DEBILIDADES & ESTRATEGIAS DO & ESTRATEGIAS DA \\
\hline $\begin{array}{l}\mathbf{D}_{1} \text {.Falta de cumplimiento de los procedimientos } \\
\text { operativos apropiados para la extracción de crudos } \\
\mathbf{D}_{2} \text {.Problemas financieros que no permiten } \\
\text { contratar/adquirir bienes y servicios. } \\
\mathbf{D}_{3} \text {. Escasa capacidad de innovación en los procesos } \\
\text { de recuperación mejorada de crudos } \\
\mathbf{D}_{4} \text {. Poca investigación y desarrollo de nuevos } \\
\text { productos. } \\
\text { D. }_{5} \text { Limitado desarrollo de planes de formación del } \\
\text { personal }\end{array}$ & $\begin{array}{l}\mathbf{E}_{5} \text {. Identificar de forma eficiente los procesos claves } \\
\text { candidatos a realizarles mejoras }\left(\mathbf{D}_{1}, \mathbf{O}_{3}\right) \\
\mathbf{E}_{6} \text {. Establecer programas de entrenamiento, } \\
\text { capacitación y formación del personal }\left(\mathbf{D}_{3}, \mathbf{D}_{5}, \mathbf{O}_{2}, \mathbf{O}_{4}\right) \\
\mathbf{E}_{7} \text {. Realizar actividades de } \mathrm{I} \mathrm{D} \text { en alianza con las } \\
\text { universidades a fin de explorar nuevas ideas } \\
\text { innovadoras }\left(\mathbf{D}_{4}, \mathbf{O}_{2}\right)\end{array}$ & $\begin{array}{l}\mathbf{E}_{10 .} \text { Establecer políticas de beneficios que } \\
\text { impulsen a mejorar la satisfacción del empleado ( } \\
\mathbf{D}_{5,} \mathbf{A}_{2} \text { ) } \\
\mathbf{E}_{11 .} \text { Gestionar de forma eficiente los recursos } \\
\text { minimizando el costo de operación }\left(D_{1}, A_{3}\right) \\
E_{12 .} \text { Consolidar la relación empresa-proveedor, a fin } \\
\text { de alcanzar una mayor especialización tecnológica } \\
\text { en los procesos de innovación } \quad\left(D_{2}, A_{1}\right)\end{array}$ \\
\hline
\end{tabular}

Fuente: Elaboración propia (2020) 
Sistema de benchmarking como estrategia de innovación tecnológica en el sector crudos pesados y extrapesados

Figura 3. Mapa estratégico.

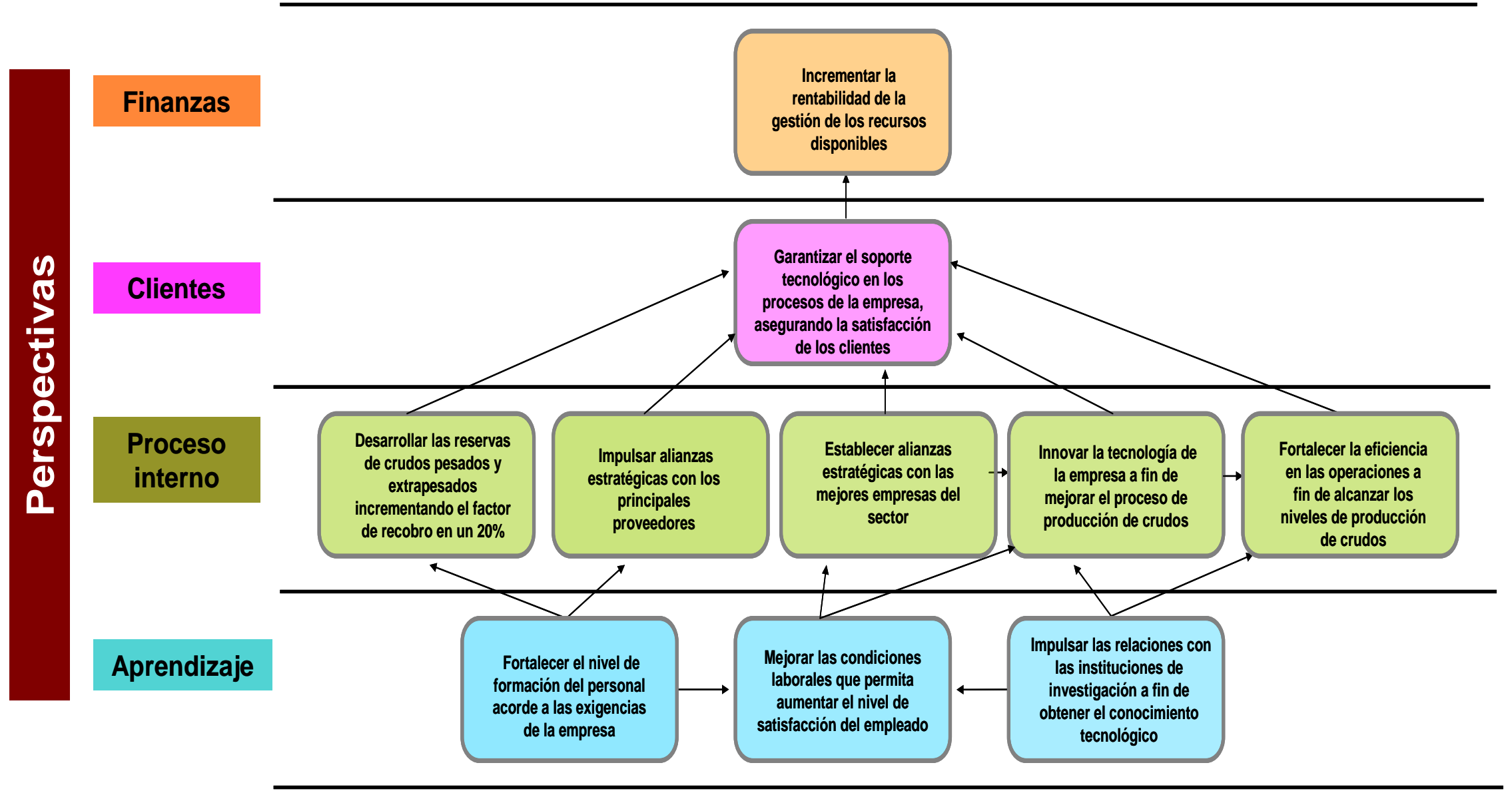

Fuente: Elaboración propia (2020).

Tabla 6. Cuadro de Mando Integral - medidas 
Sistema de benchmarking como estrategia de innovación tecnológica en el sector crudos pesados y extrapesados

\begin{tabular}{|c|c|c|c|c|c|c|c|}
\hline \multirow[b]{2}{*}{ Perspectiva } & \multirow[b]{2}{*}{ Objetivos estratégicos } & \multirow[b]{2}{*}{$\begin{array}{l}\text { Áreas } \\
\text { críticas }\end{array}$} & \multirow[b]{2}{*}{ Factores claves } & \multicolumn{4}{|c|}{ MEDIDAS } \\
\hline & & & & Indicadores & Definición operacional & $\begin{array}{l}\text { Frecuencia de } \\
\text { actuación }\end{array}$ & Fuente de datos \\
\hline Finanzas & 1. Incrementar la rentabilidad de la gestión de los recursos disponibles & Financiera & Rentabilidad & $\begin{array}{l}\text { Utilidad Neta en } \\
\text { ventas }\end{array}$ & $\begin{array}{l}\text { \%Utilidad Neta/ } \\
\text { Patrimonio }\end{array}$ & Anual & Balances financieros \\
\hline Cliente & $\begin{array}{l}\text { 2. Garantizar el soporte tecnológico en los procesos de la empresa, asegurando la } \\
\text { satisfacción de los clientes }\end{array}$ & $\begin{array}{l}\text { Marketing y } \\
\text { ventas }\end{array}$ & $\begin{array}{l}\text { Satisfacción del } \\
\text { cliente }\end{array}$ & $\begin{array}{l}\text { Satisfacción del } \\
\text { cliente }\end{array}$ & $\begin{array}{l}\% \text { de clientes satisfechos con } \\
\text { el servicio }\end{array}$ & Anual & Encuesta \\
\hline \multirow{4}{*}{$\begin{array}{r}\text { Proceso } \\
\text { interno }\end{array}$} & $\begin{array}{l}\text { 3. Desarrollar las reservas de crudos pesados y extrapesados incrementando el factor de } \\
\text { recobro en un } 20 \%\end{array}$ & \multirow{4}{*}{ Planficación } & Producción & $\begin{array}{l}\text { Incremento del } \\
\text { factor de recobro }\end{array}$ & $\begin{array}{c}\text { \%Petróleo } \\
\text { producido/POES*100 }\end{array}$ & Anual & Reportes de producción \\
\hline & $\begin{array}{l}\text { 4. Impulsar alianzas estratégicas con los principales proveedores } \\
\text { 5. Establecer alianzas estratégicas con las mejores empresas del sector }\end{array}$ & & $\begin{array}{l}\text { Alianzas } \\
\text { estratégicas }\end{array}$ & $\begin{array}{l}\text { Alianzas } \\
\text { estratégicas }\end{array}$ & $\begin{array}{l}\text { \#Alianzas estratégicas } \\
\text { concretadas }\end{array}$ & Anual & $\begin{array}{l}\text { Base de producción } \\
\text { empresarial }\end{array}$ \\
\hline & $\begin{array}{l}\text { 6. Innovar la tecnologia de la empresa a fin de mejorar el proceso de producción de } \\
\text { crudos }\end{array}$ & & Tecnología & $\begin{array}{l}\text { Innovación } \\
\text { tecnológica }\end{array}$ & $\begin{array}{c}\% \text { actualización de tecnologias } \\
\text { realizadas } / \% \text { total } \\
\text { planificadas }\end{array}$ & Trimestral & $\begin{array}{l}\text { Informe de } \\
\text { actualización }\end{array}$ \\
\hline & $\begin{array}{l}\text { 7. Fortalecer la eficiencia en las operaciones a fin de alcanzar los niveles de producción } \\
\text { de crudos }\end{array}$ & & Operaciones & Operaciones & Mejoramiento del crudo ( $\left.{ }^{\circ} \mathrm{API}\right)$ & Trimestral & Informe de operaciones \\
\hline \multirow{3}{*}{ Aprendizaje } & 8. Fortalecer el nivel de formación del personal acorde a las exigencias de la empresa & \multirow{3}{*}{$\begin{array}{l}\text { Talento } \\
\text { humano }\end{array}$} & \multirow{2}{*}{$\begin{array}{c}\text { Satisfacción del } \\
\text { empleado }\end{array}$} & Capacitación técnica & $\begin{array}{l}\% \text { capacitaciones técnicas } \\
\text { dictadas/\%capacitaciones } \\
\text { técnicas planificadas }\end{array}$ & Anual & Plan de capacitación \\
\hline & $\begin{array}{l}\text { 9. Mejorar las condiciones laborales que permita aumentar el nivel de satisfacción del } \\
\text { empleado }\end{array}$ & & & $\begin{array}{l}\text { Satisfacción del } \\
\text { empleado }\end{array}$ & $\begin{array}{c}\text { \% nivel de satisfacción del } \\
\text { empleado/\% nivel satisfacción } \\
\text { de periodos anteriores }\end{array}$ & Anual & Encuesta \\
\hline & $\begin{array}{l}\text { 10. Impulsar las relaciones con las instituciones de investigación a fin de obtener el } \\
\text { conocimiento tecnológico }\end{array}$ & & $\begin{array}{l}\text { Alianzas } \\
\text { estratégicas }\end{array}$ & $\begin{array}{l}\text { Alianzas } \\
\text { estratégicas }\end{array}$ & $\begin{array}{l}\text { \#Alianzas estratégicas } \\
\text { concretadas }\end{array}$ & Anual & $\begin{array}{l}\text { Base de producción } \\
\text { empresarial }\end{array}$ \\
\hline
\end{tabular}

Fuente: Elaboración propia (2020). 
Sistema de benchmarking como estrategia de innovación tecnológica en el sector crudos pesados y extrapesados

Tabla 7. Cuadro de Mando Integral - metas-medios

\begin{tabular}{|c|c|c|c|c|c|c|}
\hline & \multirow{2}{*}{\multicolumn{2}{|c|}{ MEDIOS }} & \multirow{2}{*}{\multicolumn{3}{|c|}{ META }} \\
\hline \multirow[b]{2}{*}{ Perspectiva } & \multirow[b]{2}{*}{ Objetivos estratégicos } & & & & & \\
\hline & & Iniciativa estratégica/proyectos & $\begin{array}{c}\text { Lider de } \\
\text { implementación }\end{array}$ & $\begin{array}{l}\text { Corto plazo } \\
\text { (hasta } 1 \text { año) }\end{array}$ & \begin{tabular}{|} 
Mediano plazo \\
(De 1 a 5 años)
\end{tabular} & $\begin{array}{l}\text { Largo plazo } \\
\text { (hasta } 10 \text { años) }\end{array}$ \\
\hline Finanzas & 1. Incrementar la rentabilidad de la gestión de los recursos disponibles & $\begin{array}{l}\text { Plan de comercialización de crudos } \\
\text { pesados y extrapesados }\end{array}$ & $\begin{array}{l}\text { Responsable } \\
\text { financiero }\end{array}$ & $25 \%$ & & \\
\hline Cliente & $\begin{array}{l}\text { 2. Garantizar el soporte tecnológico en los procesos de la empresa, asegurando la } \\
\text { satisfacción de los clientes }\end{array}$ & Plan de Marketing y ventas & $\begin{array}{c}\text { Responsable de } \\
\text { marketing y ventas }\end{array}$ & & $15 \%$ & \\
\hline \multirow{5}{*}{$\begin{array}{c}\text { Proceso } \\
\text { interno }\end{array}$} & $\begin{array}{l}\text { 3. Desarrollar las reservas de crudos pesados y extrapesados incrementando el factor de } \\
\text { recobro en un } 20 \%\end{array}$ & $\begin{array}{l}\text { Plan de recuperación de la } \\
\text { producción petrolera }\end{array}$ & $\begin{array}{l}\text { Responsable de } \\
\text { Producción }\end{array}$ & & & $25 \%$ \\
\hline & 4. Impulsar alianzas estratégicas con los principales proveedores & \multirow{2}{*}{$\begin{array}{l}\text { Programa de alianzas estratégicas } \\
\text { con agentes externos }\end{array}$} & \multirow{2}{*}{$\begin{array}{l}\text { Responsable de } \\
\text { planificación }\end{array}$} & & $20 \%$ & \\
\hline & 5. Establecer alianzas estratégicas con las mejores empresas del sector & & & & $20 \%$ & \\
\hline & $\begin{array}{l}\text { 6. Innovar la tecnologia de la empresa a fin de mejorar el proceso de producción de } \\
\text { crudos }\end{array}$ & Plan de innovación tecnológica & $\begin{array}{l}\text { Responsable de } \\
\text { tecnología }\end{array}$ & & & $50 \%$ \\
\hline & $\begin{array}{l}\text { 7. Fortalecer la eficiencia en las operaciones a fin de alcanzar los niveles de producción } \\
\text { de crudos }\end{array}$ & Programa mejoramiento de crudos & $\begin{array}{l}\text { Responsable de } \\
\text { Operaciones }\end{array}$ & & & $50 \%$ \\
\hline \multirow{3}{*}{ Aprendizaje } & 8. Fortalecer el nivel de formación del personal acorde a las exigencias de la empresa & \multirow{2}{*}{$\begin{array}{l}\text { Plan de capacitación y motivación } \\
\text { del personal }\end{array}$} & \multirow{2}{*}{$\begin{array}{l}\text { Responsable de } \\
\text { talento humano }\end{array}$} & $25 \%$ & & \\
\hline & $\begin{array}{l}\text { 9. Mejorar las condiciones laborales que permita aumentar el nivel de satisfacción del } \\
\text { empleado }\end{array}$ & & & $25 \%$ & & \\
\hline & $\begin{array}{l}\text { 10. Impulsar las relaciones con las instituciones de investigación a fin de obtener el } \\
\text { conocimiento tecnológico }\end{array}$ & $\begin{array}{c}\text { Plan de alianzas estratégicas con } \\
\text { agentes externos }\end{array}$ & $\begin{array}{l}\text { Responsable de } \\
\text { planificación }\end{array}$ & & $20 \%$ & \\
\hline
\end{tabular}

Fuente: Elaboración propia (2020) 
Presupuesto de los proyectos

Una vez elaborados los siete (07) proyectos como resultado del análisis de los objetivos estratégicos en la presente investigación, así como sus perfiles, sus actividades, fue necesario establecer bajo ciertas premisas de cálculo, los presupuestos estimados de los proyectos. A continuación, en la tabla 8, se detallan los presupuestos para cada proyecto así como su tiempo de ejecución:

Tabla 8. Presupuesto estimados de los proyectos

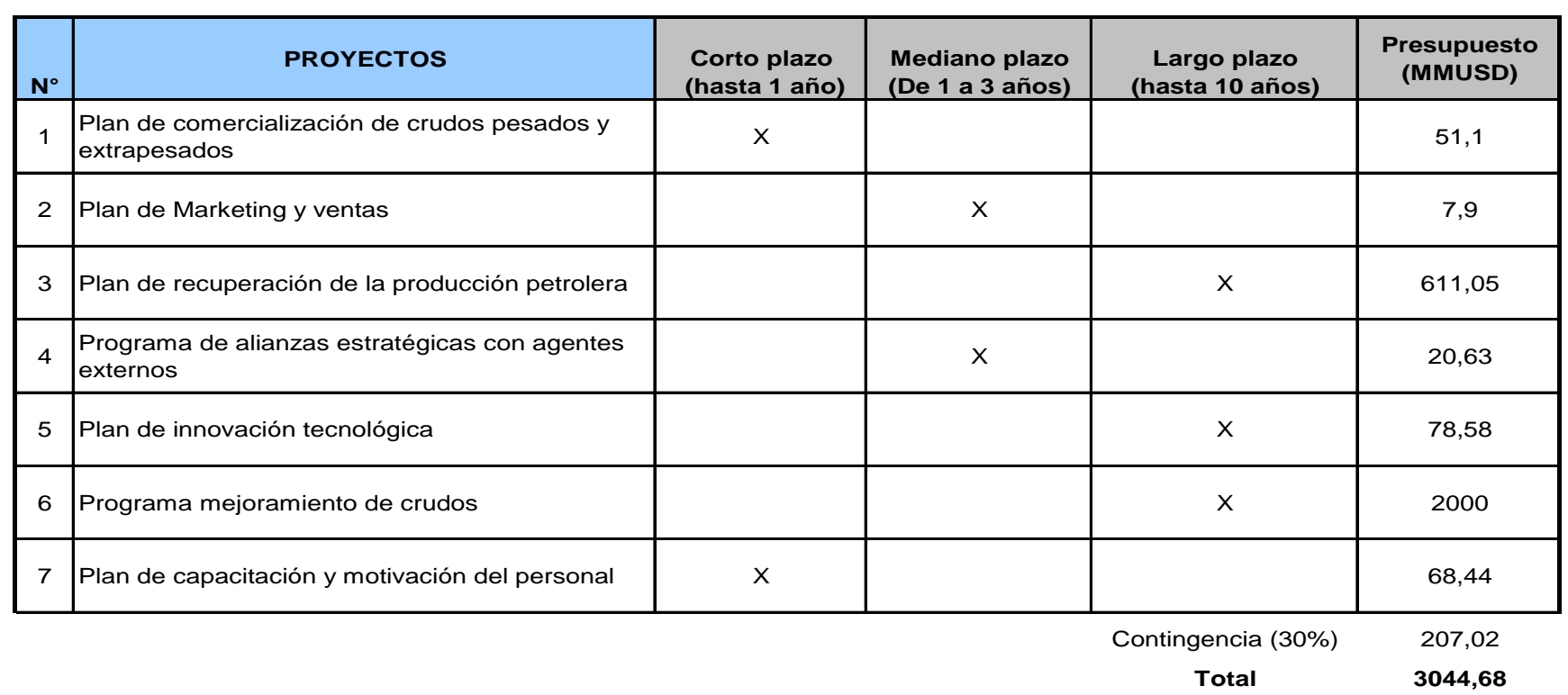

Fuente: Elaboración propia (2020).

Premisas presupuesto estimado:

1. Plan de recuperación de la producción petrolera (incluye perforación): MMUSD 611,05

a) Producción de petróleo estimada: $120 \mathrm{MBD}$

b) Costo de producción: 11 USD/Bls

c) Cantidad de servicios a pozos estimados: 300 Pozos (categoría 2) y 30 pozos a perforar.

d) Taladros, mudanzas, fluidos, cementación, cañoneo, entre otros.

e) Profundidad del pozo estimada: 2000 pies

2. Plan de innovación tecnológica: MMUSD 78,58

a) Se considero $25 \%$ en el plan de producción, por encima de las tecnologías empleadas en yacimientos convencionales, lo cual permitiría:

--Implementar tecnologías de recuperación mejorada de crudos como: Inyección alterna de vapor, calentadores eléctricos, entre otras.

- Adquisición de equipos y software de última tecnología.

- Se darían soluciones tecnológicas con INTEVEP 
Sistema de benchmarking como estrategia de innovación tecnológica en el sector

crudos pesados y extrapesados

3. Plan de marketing y ventas: MMUSD 7,9

a) Se estimo el $10 \%$ de la tecnología asociada al plan de producción, lo cual permitiría garantizar el volumen del crudo en tiempo y especificación

4. Plan de comercialización de crudos pesados y extrapesados: MMUSD 51,1

a) Producción de petróleo estimada: 120MBD

b) Traslado de un barril: 2USD

5. Programa de alianzas con agentes externos (proveedores, competidores y universidades): MMUSD 20,63

a) Se estimo $15 \%$ de la tecnología asociada al plan de producción, para las alianzas con empresas, un $60 \%$ asociado a dichas alianzas para los acuerdos con proveedores y un $10 \%$ para las alianzas con las universidades Mediante las alianzas estratégicas se lograría:

- Impulsar las alianzas con los clientes a fin de garantizar el volumen del crudo en tiempo y especificación.

- Impulsar alianzas con empresas proveedoras de bienes y servicios y tecnología.

- Impulsar alianzas con universidades de investigación, lo cual incrementaría las actividades de I+D.

- Se establecerían redes de cooperación con empresas del mismo sector, a fin de aprender de sus procesos

6. Plan de capacitación y motivación del personal: MMUSD 68,44

a) Cantidad de empleados: 1500 trabajadores. Para el plan de capacitación del personal se lograría:

-Crear un sistema de formación del personal especializado en crudos pesados y extrapesados.

-Crear un sistema de desempeño del empleado, a fin de elevar la eficiencia y eficacia en los procesos.

-Condiciones socioeconómicas favorables, a fin de lograr la satisfacción del empleado

7. Programa mejoramiento de crudos: MMUSD 2000,00

a) Se estimo reactivar el mejorador de crudos extrapesados, en vista de que actualmente se encuentra paralizado por fallas en equipos. Con esto se lograría:

- Reactivar en un 50\% el mejorador de crudos extrapesados que actualmente se encuentra paralizado.

- Una capacidad para el mejoramiento de 150 bls de $22^{\circ}$ API

Contigencia (30\%), por considerarse un estimado clase V.

Análisis de riesgos

IPSA SCIENTIA - Vol. 5 No $^{\circ} 1-2020$ 
La identificación de riesgos en un proyecto, permite determinar como estos pueden afectar al mismo. En este sentido, esta identificación se lleva cabo a través de un proceso participativo en el que el equipo del proyecto junto a los expertos en la materia u otras partes interesadas involucradas de alguna manera contribuyen con ideas y aportan su experiencia, Siles y Mondelo (2012). Sobre lo anteriormente planteado, para los siete (07) proyectos elaborados la presente investigación para la empresa mixta C objeto de estudio, se llevó a cabo la identificación de siete (07) riesgos que podrían estar presentes en cada uno de estos proyectos generados. En este sentido, se procedió a determinar su probabilidad, efecto que causan, el máximo impacto, así como la medida de mitigación, de acuerdo a lo mostrado en la tabla 9, se observa que existe el $57 \%$ de probabilidad de que los riesgos sean moderados en su impacto.

Tabla 9. Análisis de riesgos de los proyectos.

\begin{tabular}{|c|c|c|c|c|c|}
\hline $\mathrm{N}^{\circ}$ & Descripción del riesgo & Probabilidad & Efecto & Impacto & Medida de mitigación \\
\hline 1 & Bajo precios del crudo & Probable & Baja calidad del proyecto & Mayor & $\begin{array}{l}\text { Maximixar esfuerzos para } \\
\text { aumentar la producción }\end{array}$ \\
\hline 2 & Imposibilidad de cumplir con la demanda del cliente & Moderada & $\begin{array}{l}\text { Imposibilidad de llevar a cabo el } \\
\text { proyecto }\end{array}$ & Moderado & Establecer compromisos \\
\hline 3 & Dificultad para aumentar el factor de recobro del petróleo & Moderada & $\begin{array}{l}\text { Incongruencia en resultados } \\
\text { esperados }\end{array}$ & Moderado & $\begin{array}{l}\text { Aplicación de nuevas } \\
\text { tecnologias }\end{array}$ \\
\hline 4 & $\begin{array}{l}\text { Desinterés por parte de los agentes externos en llevar acabo las } \\
\text { alianzas estratégicas }\end{array}$ & Probable & $\begin{array}{l}\text { Incongruencia en resultados } \\
\text { esperados }\end{array}$ & Mayor & $\begin{array}{l}\text { Impulsar las alianzas } \\
\text { estratégicas }\end{array}$ \\
\hline 5 & $\begin{array}{l}\text { Dificultad para la definición de estrategias que permitan llevar a cabo } \\
\text { tecnologías en la empresa }\end{array}$ & Casi segura & Descoordinación en el proyecto & Moderado & Estrategias en equipo \\
\hline 6 & Imposibilidad de realizar inversión en los mejoradores de crudos & Moderada & $\begin{array}{l}\text { Imposibilidad de llevar a cabo el } \\
\text { proyecto }\end{array}$ & Moderado & $\begin{array}{l}\text { Convenios para mejorar } \\
\text { crudo en el exterior }\end{array}$ \\
\hline 7 & $\begin{array}{l}\text { Dificultad para ubicar talento humano en la empresa con } \\
\text { competencias para el desarrollo del proyecto }\end{array}$ & Moderada & Baja calidad del proyecto & Moderado & Capacitación del personal \\
\hline
\end{tabular}

Fuente: Elaboración propia (2020).

\section{Conclusiones}

Se observó presencia de las etapas del benchmarking, planificación, análisis, integración y acción; sin embargo, se evidencia el poco interés por parte de las empresas mixtas del sector crudos pesados y extrapesados, para determinar las brechas existentes con las empresas de la competencia. 
Se evidenció presencia de los cuatros tipos de benchmarking (interno, competitivo, genérico y funcional); sin embargo, se observa poca iniciativa por parte de la empresa mixta del sector curdo pesado y extrapesado de llevar a cabo un benchmarking.

Se valida la presencia del factor tamaño de la empresa. Por su parte, los factores nivel de formación, secreto industrial, redes de cooperación y obstáculos a la innovación reportan mediana presencia, lo que pone de manifiesto deficiencias asociadas a un plan de formación del personal que permita fortalecer el conocimiento en la empresa. Así como bajos niveles de apropiación de la innovación y poca disponibilidad por parte de la empresa mixta de llevar acabo alianzas de estratégicas con otros agentes industriales y agentes tecnológicos.

Se valida muy presente el indicador fuentes comerciales. Por su parte, las fuentes institucionales, información general y actividades $\mathrm{I}+\mathrm{D}$, reportan mediana presencia. Lo que indica debilidades para impulsar alianzas con las universidades, poco acceso de las empresas al conocimiento mediante revistas, base de datos de patentes y conferencias y falta de capacidad para generar el conocimiento interno

La implementación del plan estratégico basado en el benchmarking como estrategia de innovación tecnológica, mediante la técnica del cuadro de mando integral proporcionará a las empresas mixtas del sector crudos pesados y extrapesados una herramienta que le permita cumplir con sus objetivos a través del seguimiento y medición de su desempeño.

En la comparación de las empresas mixtas (benchmarking competitivo), se pudo observar que existe una brecha entre la empresa mixta C y las empresas mixtas A y B en casi el 59\% de los indicadores, principalmente en la planificación, análisis, integración, acción, benchmarking funcional, secreto industrial, redes de cooperación, actividades de I+D y fuentes comerciales.

El resultado del análisis realizado al factor externo a través de la utilización de la Matriz de Evaluación de Factores Externos (EFE), indica que las oportunidades que ofrece el sector crudos pesados y extrapesados están siendo aprovechadas medianamente. Con respecto al análisis del factor interno por medio de la Matriz de Evaluación de Factores Interno (EFI), las fortalezas representan un posicionamiento interno muy débil.

El cuadro de mando integral es un instrumento de gestión que permite la implantación de la estrategia de la empresa de una forma eficiente, debido a que proporciona la estructura adecuada para comunicar la misión y la estrategia en objetivos e indicadores organizados en cuatro perspectivas: finanzas, clientes, procesos internos y aprendizaje

Los indicadores seleccionados aplicados para el análisis del sector crudos pesados y extrapesados, permitirán hacer seguimiento a los objetivos estratégicos definidos.

Esta investigación permitió evidenciar la importancia de la relación que existe entre las variables benchmarking y la estrategia de innovación tecnológica, por lo cual se debería analizar la situación actual que presenta la empresa mixta y considerar el plan estratégico propuesto a fin de

IPSA SCIENTIA - Vol. $5 N^{\circ} 1-2020$ 
consolidar las estrategias de innovación tecnológica mediante la aplicación de un benchmarking de tipo competitivo.

Los objetivos estratégicos propuestos en el presente trabajo, plantean acciones para establecer alianzas estratégicas con las mejores empresas del sector, universidades, proveedores, clientes, así como incrementar las reservas de crudos pesados y extrapesados, innovar la tecnología dentro de la empresa, mejorar las condiciones del personal e incrementar la rentabilidad de la empresa, todo esto con el fin de desarrollar procesos de innovación exitosos.

Finalmente, se destaca la necesidad de establecer inversiones en tecnologías en los procesos de recuperación mejorada que permita aumentar el factor de recobro de los crudos pesados y extrapesados, debido a que estos procesos demuestran deficiencias en la empresa mixta objeto de estudio, además resultan fundamentales para lograr un sector empresarial competitivo que responda efectivamente a las exigencias de la industria petrolera y que permita las mejoras de las condiciones socioeconómicas del país.

\section{Referencias}

Abad, P. (2019). El Cuadro de Mando Integral Aplicado a Planificación Estratégica de la Banca Privada, Revista Ciencia UNEMI, Vol. 12 (29), 20 - 35. doi:10.29076/2528-7737.

Aponte, G. (2015). El proceso de gestión de innovación tecnológica: sus etapas e indicadores relacionados. Revista Venezolana de Análisis de Coyuntura, vol. XXI (1), 59- 90.

Baptista, D. (2012). Diseño, desarrollo y validación de una metodología para el análisis de competitividad en sectores industriales venezolanos basada en la técnica multicriterio Analytic Network Process (ANP). (tesis doctoral). Universidad Politécnica de Valencia, España.

Benavides, E. et al. (2018). Estudio de benchmarking competitivo aplicado a cuatro empresas del sector avícola colombiano. Revista Libre Empresa, vol 15(1), 9-28. doi:10.18041/1657-2815.

Bocángel, G y Bocángel, G. (2018). Un sistema de benchmarking de eficacia educativa en la gestión de procesos universitarios. Revista Investigación y Postgrado, Vol. 33(2), 167-177.

Hernández, C \& Cano, M. (2017). La importancia del benchmarking como herramienta para incrementar la calidad en el servicio en las organizaciones. EDG Virtual. Recuperado de http://biblioteca.udgvirtual.udg.mx/jspui/handle/123456789/3577

Hernández, R., Fernández, C., \& Baptista, P. (2014). Metodología de la Investigación. México D.F:: McGraw-Hill/ Interamenricana Editores S.A. de C.V.

Kaplan, R. \& Norton, D. (2000). Cuadro de mando integral. Barcelona, España: Ediciones Gestión 2000, S.A.

IPSA SCIENTIA - Vol. 5 No $^{\circ}-2020$ 
Marcillo, R \& Garzón, J (2014), Diseño de un Modelo de Control Estratégico aplicando la metodología del Cuadro de mando Integral para el intermediario de Seguros Aeroseguros Cía. Ltda. (tesis de maestría) Universidad de las Fuerzas Armadas (ESPE) Sangolquí, Ecuador.

Marciniak, R. (2017). El benchmarking como herramienta de mejora de la calidad de la educación universitaria virtual. Ejemplo de una experiencia polaca. Revista Educar, Vol. 53 (1), 171-207.

Mencias, N., \& Rodríguez, G. (2017). Gerencia estratégica tecnológica basada en benchmarking en la industria petrolera venezolana, región occidente. REVECITEC, Vol 7(1), 1-17. Recuperado de http://ojs.urbe.edu/index.php/revecitec/article/view/863

Linares-Morales, J., \& Geizzelez-Luzardo, M. (2016). Políticas científicas y tecnológicas para la gestión del conocimiento en instituciones universitarias. IPSA SCIENTIA: Revista Científica $\begin{array}{llll}\text { Multidisciplinaria, } & 1(1), & \text { Recuperado } & \text { de }\end{array}$ https://latinjournal.org/index.php/ipsa/article/view/889

Organización de Países Exportadores de Petróleo. (2020). Proven crude oil reserves. Recuperado de. http://www.opec.org.

Paternina-Pedroza, H., Linares-Morales, J. \& Hernandez-Ayala, K. (2019). Transferencia de tecnología y conocimiento en el sector exportador de bovinos. IPSA SCIENTIA: Revista Científica Multidisciplinaria, 4(1), 10-20. Recuperado a partir de https://latinjournal.org/index.php/ipsa/article/view/936

Petróleos de Venezuela, S.A. (2017). Informe de Comisario de PDVSA. Recuperado de www.pdvsa.com.

Pérez, E. (2019). Modelo de desarrollo y política petrolera en Venezuela en el contexto de la geopolítica petrolera internacional: hacia una propuesta de plan estratégico de desarrollo. Periodo 1998 - 2018, Maracaibo, Venezuela.

Siles \& Mondelo (2012). Gestión de proyectos para resultados. Guía de gestión de proyectos. 2da edición. Recuperado de http://www.issuu.com.

Tejada, J. et al. (2019). Innovación tecnológica: Reflexiones teóricas. Revista Venezolana de Gerencia. vol. 24, (85), 12. 